\title{
Incomplete Partition type I: Radiological Evaluation of the Temporal Bone
}

Safak Parlak', [MD]

ORCID: 0000-0002-6028-4653

Ayca Akgoz Karaosmanoglu', [MD]

ORCID: 0000-0001-6991-9961

Sevtap Arslan', [MD]

ORCID: 0000-0001-9880-3095

Levent Sennaroglu ${ }^{2},[\mathrm{MD}]$

ORCID: 0000-0001-8429-2431

${ }^{1}$ Hacettepe University Faculty of Medicine, Department of Radiology, Ankara, Turkey.

${ }^{2}$ Hacettepe University Faculty of Medicine, Department of Otolaryngology, Ankara, Turkey.

\section{Corresponding Author: Safak Parlak}

Hacettepe University Faculty of Medicine Department of Radiology, Sinhiye, Ankara, Turkey.

E-mail: parlaksafak@gmail.com

https://doi.org/10.32552/2021.ActaMedica.683

\section{n ABSTRACT Con}

Objective: Incomplete partition type I is an uncommon congenital anomaly of the inner ear, characterized by typical cystic cochleovestibular appearance. Incomplete partition type I was firstly defined as cystic cochlea and vestibule without large vestibular aqueduct; however, large vestibular aqueduct and/or enlarged endolymphatic duct could rarely be seen in incomplete partition type I anomaly. Correct diagnosis of the type of cochlear malformation and differentiation of incomplete partition type $I$ is necessary for patient management and surgical approach. Our aim was to document the temporal bone imaging findings in a series of patients with incomplete partition type I.

Materials and Methods: CT ( $n=85)$ and/or MRI $(n=80)$ examinations of 99 ears in 59 incomplete partition type I patients were retrospectively evaluated. All structures of the otic capsule were retrospectively assessed. The appearances of cochlea and vestibule, vestibular aqueduct/endolymphatic duct, semicircular canals were qualitatively evaluated by an experienced neuroradiologist. The vertical dimension of vestibular aqueduct and/or endolymphatic duct (from the point where the duct arises from the vestibule) was measured on CT/MRI. Anteriorposterior diameter of the internal acoustic canal and the diameter of cochlear aperture were measured on CT. The cochleovestibular nerves were evaluated on sagittal-oblique high T2-weighted imaging.

Results: All 99 ears had defective partition with unpartitioned cochlear basal turn and absent interscalar septae, separated but cystic cochlea. The vestibule was enlarged in all ears except one. Semicircular canals were usually dysplastic (92.9\%). A total of 35 incomplete partition type I ears (35.3\%) had large vestibular aqueduct and/or enlarged endolymphatic duct. Internal acoustic canal was wide in $21 \%$ of ears. Cochlear aperture was wide in $5.9 \%$ of ears. Cochlear nerve was either hypoplastic or aplastic in about a quarter of incomplete partition type l ears.

Conclusion: In up to one-third of incomplete partition type I patients, an associated large vestibular aqueduct /endolymphatic duct could be seen accompanying typical inner ear findings. Although the cochlear nerves are normal in the majority of cases, auditory brainstem implantation may be necessary in certain cases of incomplete partition type I anomaly.

Keywords: Sensorineural hearing loss, computed tomography, incomplete partition type I anomaly, magnetic resonance imaging, large vestibular aqueduct

Received: 6 September 2021, Accepted: 21 October 2021,

Published online: 20 November 2021 


\section{INTRODUCTION}

Incomplete partition type I (IP-I) is a form of congenital cochlear partition anomalies, presenting with sensorineural hearing loss (SNHL). IP-I has descriptive imaging findings including absence of bony modiolus and cribriform plate between the base of the cochlea and internal auditory canal (IAC) and the absence of the interscalar septae (ISS) leading to a cystic appearance of the cochlea [1-3]. IP-I is also known as cystic cochleovestibular malformation since dilated vestibule is one of the usual findings [3].

The other structures of the otic capsule may also be affected. Semicircular canals (SCCs) can be either normal or dysplastic $[1,4,5]$ and IAC can be normal, enlarged, or atretic [6]. The vestibular aqueduct is usually normal $[1,3]$. However, large vestibular aqueduct (LVA) in IP-I anomaly was also reported $[1,4,7]$.

Magnetic resonance imaging (MRI) enables cochleovestibular nerve imaging [8]. Patients with IP-I may have intact cochleovestibular nerves and cochlear implantation is a treatment method in IP-I, though with high risks of cerebrospinal fluid (CSF) gusher and facial nerve injury $[4,5,9,10]$. Correct diagnosis of cochleovestibular malformation is necessary to choose the most appropriate surgical approach, selection of electrode type, and determining the risk of gusher before intervention $[10,11]$. In this study, we aimed to assess temporal bone imaging findings of IP-I anomaly in a series of patients. To the best of our knowledge, our cohort is the largest series in literature [4, 9].

\section{MATERIAL AND METHODS}

\section{Patients}

Our Institutional Review Board (IRB) has approved this retrospective study and informed consent has been waived by IRB due to the retrospective design of the study (GO 21/712).

A database search was performed from Hospital Information System by using words 'incomplete partition type I and IP-I' to identify all patients who had a temporal bone imaging scanned between January 2002 and December 2018 and who were reported to have IP-I at our tertiary hospital. All the identified cases were reassessed to check for inclusion. Inclusion criteria were i) Patients with baseline temporal bone imaging available in the PACS database, ii) typical computed tomography (CT) and/or MRI findings of cochlea demonstrating IP-I anomaly including the absence of bony modiolus and cribriform plate between the base of the cochlea and IAC and absence of the ISS leading to a cystic cochlear appearance. Exclusion criteria were patients with image distortion or artifacts.

\section{Image Acquisition}

Temporal bone CTs were obtained in the axial plane using a multidetector scanner (Somatom Plus 4/ Volume Zoom, Siemens, Erlangen, Germany), with $0.5 \mathrm{~mm}$ collimation and 0.5 to $0.6 \mathrm{~mm}$ slice thickness and sagittal and coronal reformatted images were created parallel and perpendicular to the plane of lateral SCCs for each ear, respectively.

Temporal bone MRIs were performed with either a 3T (Ingenia, Philips or Allegra, Siemens) or a 1.5T scanner (Symphony, Siemens, or Achieva Philips), by using a standard head coil. The standard temporal bone protocol included axial and sagittal oblique three-dimensional (3D) heavily $\mathrm{T} 2$ images (constructive interference in steady-state (CISS), driven equilibrium radiofrequency reset pulse (DRIVE) or fast imaging employing steady-state acquisition (FIESTA)) beside conventional axial and coronal T1-weighted imaging (WI) and T2WI. The parameters of high T2WI were as follows; CISS: FOV: 150X170, TR/TE: 7.3/3.6 ms, slice thickness: 0.6 to $1 \mathrm{~mm}$, NEX: 1.0, FA: $48^{\circ}$; FIESTA: FOV: $160 X 160$, TR/ TE: $6.3 / 2.5 \mathrm{~ms}$, slice thickness: 0.6 to $1 \mathrm{~mm}$, NEX: 1.0 to 2.0, FA: $55^{\circ}$ and DRIVE: FOV: $140 \times 140$, TR/TE: 1550/226 ms, slice thickness: 0.6 to $1 \mathrm{~mm}, \mathrm{NEX}: 1.0$, FA: $90^{\circ}$.

\section{Imaging Evaluation}

CT and MRI examinations were retrospectively evaluated by a neuroradiologist, 7 year-experienced in head and neck imaging.

The appearances of cochlea and vestibule, vestibular aqueduct/endolymphatic duct, SCCs, and IAC were qualitatively evaluated. The absence/ presence of ISS, modiolus, and cribriform plate were noted. External acoustic canal (EAC), middle ear cavity were also assessed for accompanying abnormality. 
Cochlear aperture, stapes, and oval window, otic capsule thickness, facial nerve canal, and presence of semicircular canal dehiscence were also assessed on CT.

The cochleovestibular nerves were evaluated on sagittal-oblique high $\mathrm{T} 2 \mathrm{Wl}$ if present. Cochlear nerve hypoplasia was defined when cochlear nerve diameter was smaller than the facial nerve diameter on sagittal-oblique high T2WI.

\section{Quantitative Evaluation}

The vertical dimensions of the vestibular aqueduct (from the point where the duct arises from the vestibule) and anterior-posterior (AP) diameter of IAC (from the midpoint of the longitudinal diameter of $I A C$ ) were measured on $C T$. The vertical dimension of the endolymphatic duct was also measured on MRI. Vestibular aqueduct dilatation was considered when the vestibular aqueduct was greater than 1 $\mathrm{mm}$ (based on the work of Vijayasekaran) [12] and endolymphatic duct dilatation was considered when the endolymphatic duct was greater than 1.4 $\mathrm{mm}$ [13].

Cochlear aperture, which is situated between the cochlear base and the fundus of the IAC was measured and classified as normal, wide, or stenotic with a range of $1.2 \mathrm{~mm}$ to $2.5 \mathrm{~mm}$ based on Lim et al study [14].

Wide IAC was considered when the AP diameter of IAC was greater than $5 \mathrm{~mm}$ in children and $4.48 \mathrm{~mm}$ in adults [15].

\section{Clinical Evaluation}

Operative reports of cases, which had undergone surgery were also retrospectively reviewed for cerebrospinal fluid (CSF) complications.

\section{Statistical Analysis}

Mean and standard deviations (SD) and median (min/max) values were given for variables with normal distribution and without normal distribution, respectively. The normality assumption was assessed by the Kolmogorov-Smirnov test. Categorical variables were given as percentages. All analyses were performed using the IBM SPSS Statistics 23.0.

\section{RESULTS}

The study consisted of 99 ears (R/L: 51/48) of 59 IP-I patients (F/M: 42/17) with contralateral IP-II in three ears, cochlear hypoplasia in eight ears, complete labyrinthine aplasia in five ears, and normal cochlea in three ears. The median age was 3 years (min: 7 months, max: 21 years). All cases were younger than 18 years old except one.

Characteristics of patients were summarized in Table 1. All patients were presented with SNHL. Twenty-seven ears of the 27 patients had a history of cochlear implantation and 10 ears had gusher (a massive flow of CSF through footplate defect or during cochleostomy) during surgery.

Table 1. Characteristics of all patients $(n=59)$ with IP-1

\begin{tabular}{|c|c|}
\hline Characteristic & \\
\hline Age, years (median, [range]) & 3 [7 months -21 years] \\
\hline Sex (F:M) & $42: 17$ \\
\hline Sensorineural hearing loss, $\mathrm{n}^{\mathrm{a}}(\%)$ & $59(100 \%)$ \\
\hline Cochlear implantation, $\mathrm{n}^{\mathrm{b}}(\%)$ & $27(45.7 \%)$ \\
\hline Gusher during surgery & $10(37 \%)$ \\
\hline Enlarged vestibule, $\mathrm{n}^{\mathrm{b}}(\%)$ & 98 (99\%) \\
\hline Dysplastic SCCs, n ${ }^{\mathrm{b}}(\%)$ & $92(92.9 \%)$ \\
\hline Total or partial aplasia & $7(7.6 \%)$ \\
\hline Dilatation in proximal part of SCCs & $23(25 \%)$ \\
\hline Other $^{c}$ & $62(67.4 \%)$ \\
\hline LVA and/or enlarged endolymphatic duct, n b (\%) & $35(35.3 \%)$ \\
\hline \multicolumn{2}{|l|}{ LVA: Large vestibular aqueduct } \\
\hline \multicolumn{2}{|l|}{ SCC: Semicircular canal } \\
\hline \multicolumn{2}{|l|}{ a Number of patients } \\
\hline${ }^{b}$ Number of ears & \\
\hline
\end{tabular}


All ears had characteristic IP-I anomaly; a cystic appearing cochlea with lack of separation of cochlear basal turn and absent ISS which can be clearly differentiated from a dilated vestibule (Figure 1). Modiolus and the cribriform plate were absent in all ears except two. In these two ears, a very thin modiolus at the cochlear base was observed.

Vestibule was enlarged in all ears except one. Ninety-two (92.9\%) ears revealed dysplastic SCCs (including shape abnormality, mild to severe dilatation, total or partial absence of any SCC or fusion abnormality of SCCs, or absence of bone islands) (Figure 2). In 7 ears of these 92 ears (7.6\%) total or partial aplasia of SCCs was present. 23 of 92 (25\%) ears revealed dilatation in the proximal part of SCCs. SCCs were normal in 7 ears (7.1\%).

A total of 35 IP-I ears (35.3\%) had LVA and/or enlarged endolymphatic duct.

Abnormal findings of ears with IP-I on crosssectional imaging were summarized in Table 2.

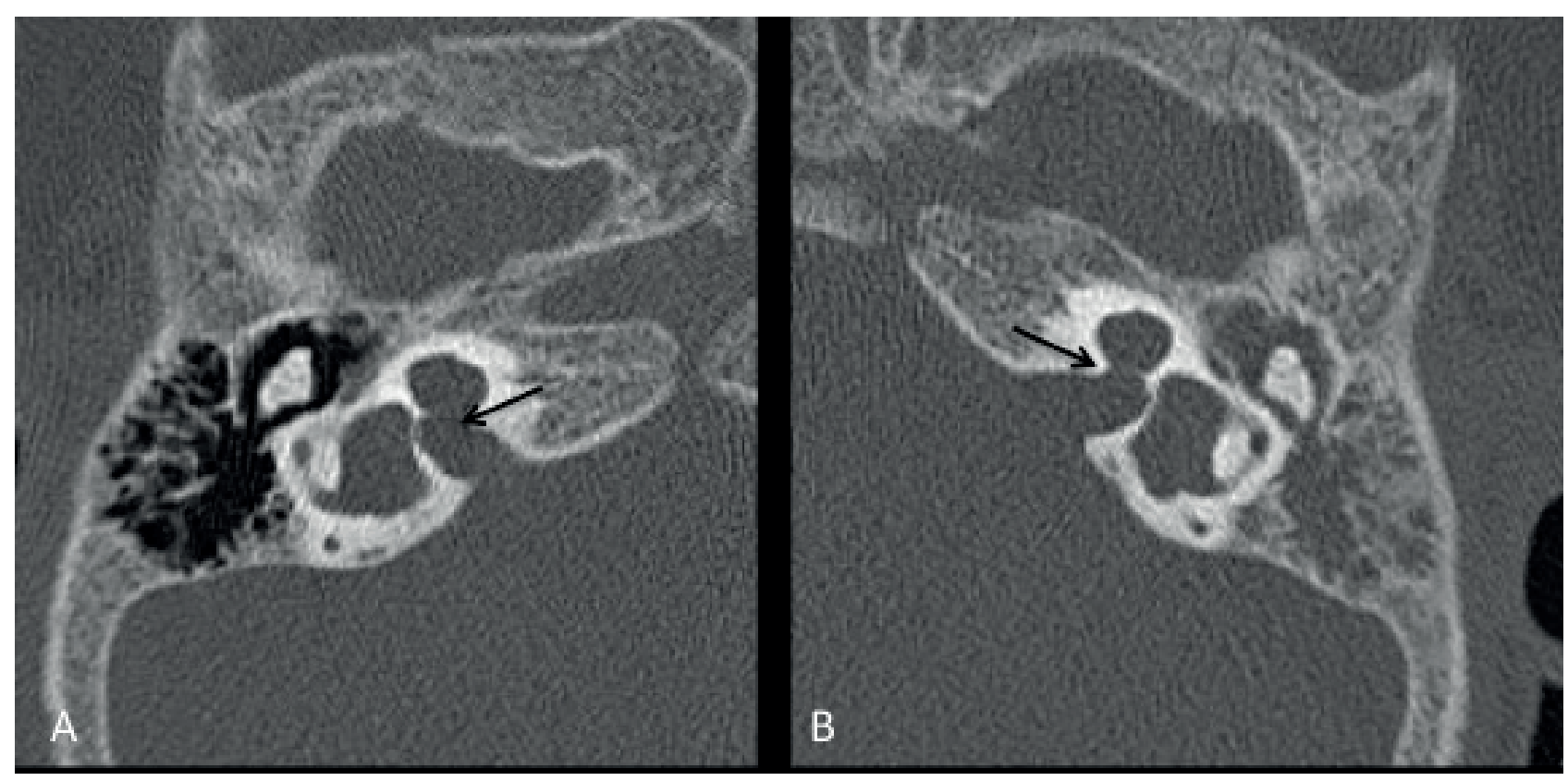

Figure 1. Axial temporal bone CT images of a 9-month girl with IP-I anomaly (Right ear (A), left ear (B)). Bilateral cystic cochleovestibular malformation with the absence of cribriform plate and modiolus, with absent interscalar septae. Notice the enlarged cochlear aperture (arrows) and the opacification filling the left middle ear cavity (B).

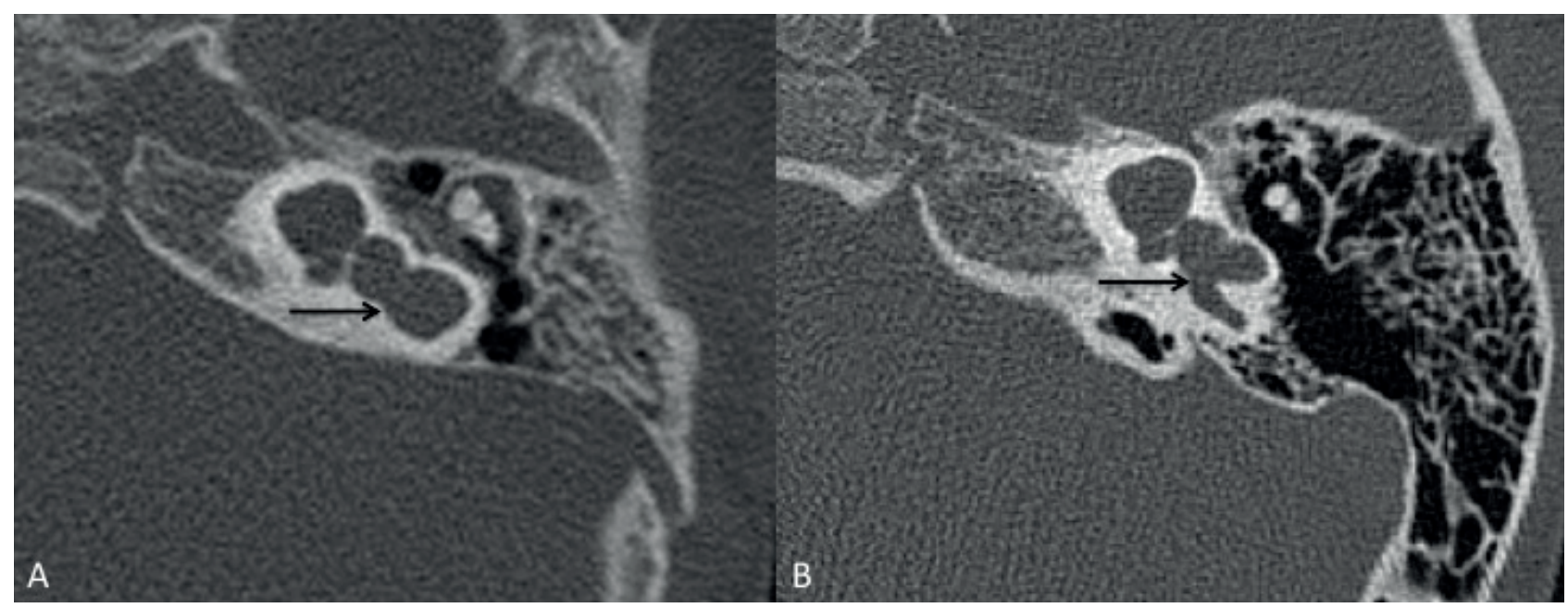

Figure 2. Examples of dysplastic vestibules and dysplastic semicircular canals in IP-I anomaly on temporal bone CT (arrows). Shape and fusion abnormality of the vestibule and SCC with dilatation and absence of bone island (A). Shape abnormality of the vestibule and SCCs, partial absence of posterior SCC, and fusion abnormality of lateral SCC with the absence of corresponding bone island. 
Table 2. Abnormal findings of ears with IP-I on cross-sectional imaging

\begin{tabular}{|c|c|}
\hline \multicolumn{2}{|l|}{$\mathrm{CT}$ findings ${ }^{\mathrm{a}}, \mathrm{n}^{\mathrm{b}}(\%)$} \\
\hline Enlarged cochlear aperture & $5(5.9 \%)$ \\
\hline Large vestibular aqueduct & $31(36 \%)$ \\
\hline Diameter of vestibular aqueduct, mm (median, [range]) & $1.5[1.1-1.4]$ \\
\hline Enlarged IAC & $18(21 \%)$ \\
\hline SCC dehiscence $^{c}$ & $5(5.9 \%)$ \\
\hline Opacification filling the middle ear cavity & $22(25.9 \%)$ \\
\hline Non-visualization of stapes & $11(13 \%)$ \\
\hline Labyrinthitis ossificans & $1(1.2 \%)$ \\
\hline \multicolumn{2}{|l|}{ MRI findings ${ }^{d}, n^{b}(\%)$} \\
\hline Enlarged endolymphatic duct & $32(40 \%)$ \\
\hline Diameter of endolymphatic duct; mm (median, [range]) & $1.8[1.5-2.8]$ \\
\hline Enlarged endolymphatic sac & $2(2.5 \%)$ \\
\hline Cochlear nerve hypoplasia & $13(16.2 \%)$ \\
\hline Cochlear nerve aplasia & $5(6.3 \%)$ \\
\hline \multicolumn{2}{|l|}{ IAC: Internal acoustic canal } \\
\hline \multicolumn{2}{|l|}{ SCC: Semicircular canal } \\
\hline \multicolumn{2}{|l|}{ a Available in 51 patients ( 85 ears) } \\
\hline \multicolumn{2}{|l|}{${ }^{\mathrm{b}}$ Number of ears } \\
\hline \multicolumn{2}{|l|}{ 'All located in superior SCC } \\
\hline d Available in 46 patients ( 80 ears) & \\
\hline
\end{tabular}

\section{CT Findings}

85 ears of 51 patients had CT examinations with contralateral IP-II in two ears, cochlear hypoplasia in eight ears, complete labyrinthine aplasia in five ears, and normal cochlea in two ears. The mean diameter of cochlear aperture was $2.1 \pm 1.41 \mathrm{~min} /$ max: 1.3/3.3)). Cochlear aperture was wide in five ears (Figure 1).

31 ears (36\%) had dilated vestibular aqueducts (bilaterally in six patients) (Figure 3). The median diameter of enlarged vestibular aqueducts was 1.5 $\mathrm{mm}$ with a range of 1.1 to $4 \mathrm{~mm}$. IAC was wide in 18 ears (21\%).

The incidence of semicircular canal dehiscence (SCCD) was $5.9 \%(n=5)$ and all were located in the superior semicircular canal. The facial nerve canal was normal in all ears.

Opacification filling the middle ear cavity was detected in 22 ears, in one MRI was not available and in the remaining 21 ears MRI revealed fluid signal intensity filling the middle ear cavity. Five of these had a history of spontaneous CSF fistula proven by surgery.

Stapes was not clearly visualized from surrounding opacifications in 11 ears. In the remaining 79 ears, stapes was normal. The width of the oval window and the depth of the oval window niche were normal qualitatively in all ears.

Otic capsule thickness was qualitatively normal in all ears. The external acoustic canal was normal in all ears.

Labyrinthitis ossificans was detected in posterior SCC in one patient.

\section{MRI Findings}

80 ears of 46 patients had MRI examinations with contralateral IP-II in one ear, cochlear hypoplasia in five ears, complete labyrinthine aplasia in five ears, and normal cochlea in one ear (69 ears had both CT and MRI). 31 patients had MRI on a 1.5T scanner and 15 patients had MRI on a 3T scanner.

32 ears (40\%) had large endolymphatic duct (bilaterally in 7 patients) with bilateral enlarged endolymphatic sac (2.5\% in ears) in one patient (Figure 3). The median diameter of the enlarged endolymphatic duct was $1.8 \mathrm{~mm}$ (min/max: 1.5/2.8 $\mathrm{mm})$.

Vestibular and facial nerves were normal in all 80 ears. 62 ears (77.5\%) had normal cochlear nerves. Cochlear nerve hypoplasia rate was $16.2 \%(n=13$ ears) in IP-I. Five ears (6.3\%) had no cochlear nerves. 

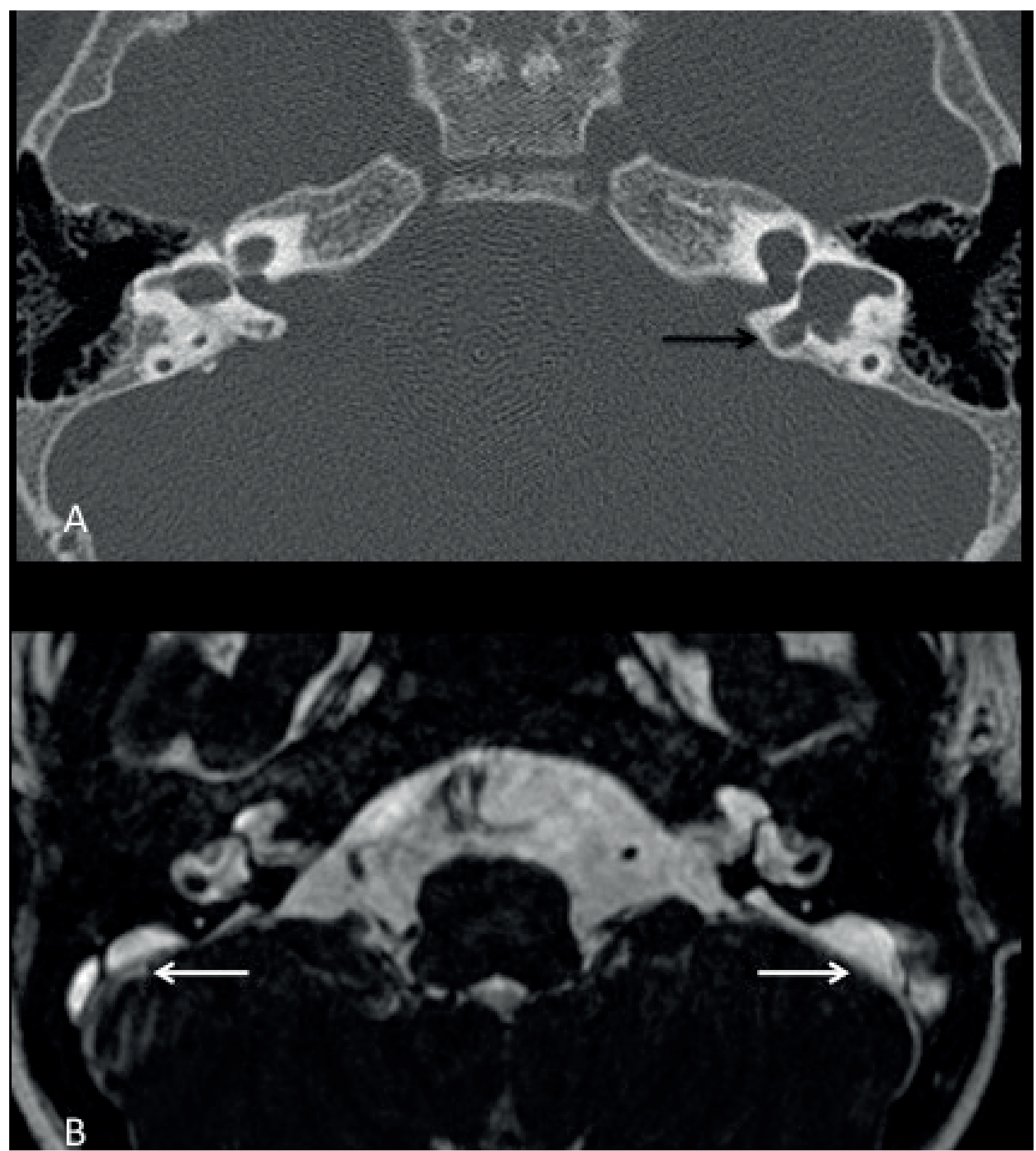

Figure 3. Enlarged vestibular aqueduct of left ear on temporal bone CT image of a 7-month-old girl with IP-I anomaly (A, black arrow). Bilateral enlarged endolymphatic duct and sac (white arrows) with IP-I anomaly on heavily T2 image of a 15-month-old boy (B).

\section{DISCUSSION}

Congenital cochleovestibular malformations occur due to the arrest of the development of embryological inner ear structures in the 3rd to 7 th gestational weeks at various stages $[1,2,16]$. Several factors including genetic abnormalities and environmental factors can affect the normal development of the membranous labyrinth [2]. Incomplete partition anomalies comprise cochlear internal architecture abnormalities, with clear differentiation of the cochlea from the vestibule. 
IP-I, one type of cochlear partition anomalies, constitutes approximately $17 \%$ of the inner ear malformations [1].

In the 1980s; Jackler et al. proposed a classification of congenital inner ear malformation with polytomography as complete labyrinthine aplasia (Michel deformity, in 3rd week), cochlear aplasia (in late 3rd week), common cavity (in 4th week), cochlear hypoplasia (in 6th week), and incomplete partition (Mondini deformity; in 7th week) [16]. Afterwards, Sennaroglu and Saatci suggested two different types of incomplete partition and proposed a new classification system based on CT and MRI findings as follows: Michel deformity, cochlear aplasia, common cavity, cystic cochleovestibular malformation (IP-I), cochlear hypoplasia, and Mondini deformity (IP-II) [1]. They defined IP-I as cystic cochlea and vestibule without LVA, and IP-II as cystic cochlear apex and dilated vestibule and LVA [ 1, 3]. However, some authors reported LVA in a few IP-I cases [5-7]. In later studies consisting relatively small cohort of IP-I cases, LVA was reported in a range of 20 to $32 \%$ in IP-I (in 24 and 41 ears respectively) $[4,9]$. A total of 35 IP-I ears (35.3\%) in our cohort had LVA and/or enlarged endolymphatic duct in line with previous studies. The enlarged endolymphatic sac can also be seen in IP-I based on our results.

The absence of cribriform plate and modiolus in IP-I is known to cause misplacement of the cochlear implant electrode into the internal auditory canal [17]. Correct diagnosis of the type of cochlear malformation and differentiation of IP-I from IP-II is necessary for patient management and determining the risk of gusher before intervention. The main features on CT of IP-I include cystic cochlea with unpartitioned basal turn and absent ISS, incomplete separation of the cochlea from the IAC due to absent bony modiolus, and cribriform plate $[1,11]$. In this anomaly, cochlear internal architecture is deficient and without bony modiolus, the cochlea seems like an empty cystic structure with a widened cochlear aperture. The basal turn of the cochlea is not clearly partitioned from the rest of the cochlea in IP-I, however, in IP-II, a partitioned basal turn is present. In, IP-II, due to defective ISS between the upper-basal and uppermiddle turns and between the lower-middle and the apical turns, the coalescence of the apical and middle turns form the cystic appearance of the cochlear apex. However, all ISS were entirely absent in the IP-I anomaly [11].

It is also very important to differentiate cochlear hypoplasia type II (CH-II) from IP-I. They look exactly similar; in $\mathrm{CH}-\mathrm{Il}$ cochlea can be differentiated clearly from the vestibule it is unpartitioned but the size of the cochlea is smaller than normal cochlea [18]. The importance is to choose a shorter electrode with stopper. If the cochlea cannot be differentiated clearly from the vestibule the anomaly is termed as common cavity. This involves a different surgical approach, which is transmastoid labyrinthotomy. In IP-I and $\mathrm{CH}-\mathrm{II}$ however, the facial recess is opened and the electrode is placed directly into the cochlea.

Various degrees of modiolar deficiency can be seen in IP-II [11]. In IP-II, developmental arrest in otic placode occurs at the 7th week and partial development of modiolus takes place [1]. Sennaroglu and Saatci reported complete absence of the modiolus with a defective cribriform plate and enlarged IAC in IP-I and suggested an earlier developmental arrest in IP-I than IP-II [1, 2]. Due to the absence of modiolus in IP-I, an earlier developmental arrest in the otic placode (in the 5th week) was suggested [1]. In a later study; the subtotal modiolar defect is defined with histopathological examination however it is not generally possible to visualize this thin modiolar base with current imaging techniques [2]. In our cohort, two ears had very thin modiolus at the cochlear base.

Sennaroglu histopathologically observed defective endosteum (inner periosteal layer of the otic capsule) with normal middle enchondral and outer periosteal layers in IP-I cases and suggested defective vascular supply [2]. Endosteum has a vascular supply from the IAC blood vessels while the middle enchondral and outer periosteal layers have vascular supply coming from the middle ear. Blood vessels from IAC supply modiolus, osseous spiral lamina, ISS. In IP-I as in our cohort, all of these structures were absent supporting Sennaroglu's theory. The otic capsule thickness was normal in all cases. Preserved otic capsule thickness can be explained with normal development of the enchondral and periosteal layers.

SCCS are generally dilated in IP-I with dysplasia in the majority and aplasia in some of the ears and 
normal SCC can also be seen, our results were in line with previous studies $[1,4,5]$. The prevalence of SCCD was not higher in our IP-I cohort than the previously reported prevalence of SCCD in the normal population $[19,20]$.

High CSF pressure and spontaneous CSF fistula and defect in the stapes footplate and thus CSF leak in IP-I cases and high risk of recurrent meningitis were previously reported $[2,5,21]$. A higher percentage of CSF gusher during surgery was previously documented [4]. Facial nerve displacement and facial nerve stimulation after cochlear implantation was also reported [22]. In our cohort, no facial nerve canal abnormality was detected. One ear revealed labyrinthine ossificans, suggestive of previous meningitis. One-fifth of the ears had middle ear opacification with reported CSF leak in $20 \%$ of these.

Cochlear nerves are normal in the majority of cases and can be either hypoplastic or aplastic in IP-I in about a quarter of IP-I ears. $[4-6,9,10]$. This finding suggests that auditory brainstem implantation may be necessary in certain cases of IP-I anomaly.
Our study had some limitations due to the retrospective design. Although temporal bone CT/ MRI protocol was standardized and include highresolution thin slice imaging, imaging parameters such as slice thickness was varied among years or vendors. Secondly, we did not compare radiologic findings with hearing test results, this may be a limitation.

In conclusion, IP-I has pathognomonic inner ear imaging findings. In IP-I anomaly, associated LVA/ enlarged endolymphatic duct can also be seen. Cochlear nerves can be either hypoplastic or aplastic in IP-I in about a quarter of IP-I ears. In this study, we aimed to assess temporal bone imaging findings of IP-I anomaly in the largest series in literature to the best of our knowledge. Our results were in line with previous studies.

\section{CONFLICT Of INTEREST}

The authors declare that they have no competing interests.

\section{weO REFEENCES Cem}

[1] Sennaroglu L, Saatci I. A new classification for cochleovestibular malformations. Laryngoscope. 2002; 112: 2230-2241.

[2] Sennaroglu L. Histopathology of inner ear malformations: Do we have enough evidence to explain pathophysiology? Cochlear Implants Int. 2016; 17: 3-20.

[3] Sennaroglu L, Saatci I. Unpartitioned versus incompletely partitioned cochleae: radiologic differentiation. Otol Neurotol. 2004; 25: 520-529.

[4] Kontorinis G, Goetz F, Giourgas A, et al. Radiological diagnosis of incomplete partition type I versus type II: significance for cochlear implantation. Eur Radiol. 2012; 22: 525-532.

[5] Berrettini S, Forli F, De Vito A, et al. Cochlear implant in incomplete partition type I. Acta Otorhinolaryngol Ital. 2013; 33: 56-62.

[6] Adibelli ZH, Isayeva L, Koc AM, et al. The new classification system for inner ear malformations: the INCAV system. Acta Otolaryngol. 2017; 137: 246-252.

[7] Baheti AD. A case of bilateral incomplete partition type I with enlarged vestibular aqueducts: an unreported entity. Clin Radiol. 2013; 68: 98-99.
[8] Casselman JW, Offeciers FE, Govaerts PJ, et al. Aplasia and hypoplasia of the vestibulocochlear nerve: diagnosis with MR imaging. Radiology. 1997; 202: 773-781.

[9] Ozbal Batuk M, Cinar BC, Ozgen B, et al. Audiological and Radiological Characteristics in Incomplete Partition Malformations. J Int Adv Otol. 2017; 13: 233-238.

[10] Sennaroglu L, Sarac S, Ergin T. Surgical results of cochlear implantation in malformed cochlea. Otol Neurotol. 2006; 27: 615-623.

[11] Sennaroglu L, Bajin MD. Classification and Current Management of Inner Ear Malformations. Balkan Med J. 2017; 34: 397-411.

[12] Vijayasekaran S, Halsted MJ, Boston M, et al. When is the vestibular aqueduct enlarged? A statistical analysis of the normative distribution of vestibular aqueduct size. AJNR Am J Neuroradiol. 2007; 28: 1133-1138.

[13] Harnsberger HR, Dahlen RT, Shelton C, et al. Advanced techniques in magnetic resonance imaging in the evaluation of the large endolymphatic duct and sac syndrome. Laryngoscope. 1995; 105: 1037-1042.

[14] Lim CH, Lim JH, Kim D, et al. Bony cochlear nerve canal stenosis in pediatric unilateral sensorineural hearing loss. Int J Pediatr Otorhinolaryngol. 2018; 106: 72-74. 
[15] Marques SR, Ajzen S, D'Ippolito G, et al. Morphometric analysis of the internal auditory canal by computed tomography imaging. Iran J Radiol. 2012; 9: 71-78.

[16] Jackler RK, Luxford WM, House WF. Congenital malformations of the inner ear: a classification based on embryogenesis. Laryngoscope. 1987; 97 (3 Pt 2 Suppl 40): 2-14.

[17] Levent Sennaroğlu, Gamze Atay, Münir Demir Bajin. A new cochlear implant electrode with a "cork"-type stopper for inner ear malformations. Auris Nasus Larynx. 2014; 41: 331-336

[18] Pamuk G, Pamuk AE, Akgöz A, et al. Radiological measurement of cochlear dimensions in cochlear hypoplasia and its effect on cochlear implant selection. J Laryngol Otol. 2021; 135: 501-507.
[19] Ceylan N, Bayraktaroglu S, Alper H, et al. CT imaging of superior semicircular canal dehiscence: added value of reformatted images. Acta Otolaryngol. 2010; 130: 9961001.

[20] Ho ML, Moonis G, Halpin CF, et al. Spectrum of Third Window Abnormalities: Semicircular Canal Dehiscence and Beyond. AJNR Am J Neuroradiol. 2017; 38: 2-9.

[21] Sennaroglu L. Cochlear implantation in inner ear malformations--a review article. Cochlear Implants Int. 2010; 11: 4-41.

[22] Eftekharian A, Eftekharian K, Mokari N, et al. Cochlear implantation in incomplete partition type I. Eur Arch Otorhinolaryngol. 2019; 276: 2763-2768. 